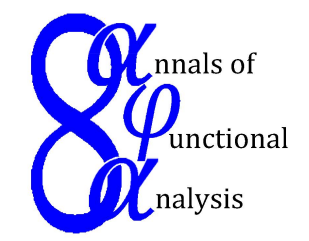

Ann. Funct. Anal. 6 (2015), no. 4, 247-254

http://doi.org/10.15352/afa/06-4-247

ISSN: 2008-8752 (electronic)

http://projecteuclid.org/afa

\title{
ON DIVERGENCE OF ANY ORDER CESÀRO MEAN OF LOTKA-VOLTERRA OPERATORS
}

\author{
MANSOOR SABUROV
}

Communicated by K. Ciesielski

\begin{abstract}
Based on some numerical calculations, S.M. Ulam has conjectured that the ergodic theorem holds true for any quadratic stochastic operator acting on the finite dimensional simplex. However, M.I. Zakharevich showed that Ulam's conjecture is false in general. Later, N.N. Ganikhodjaev and D.V. Zanin have generalized Zakharevich's example in the class of quadratic stochastic Volterra operators acting on 2D simplex. In this paper, we provide a class of Lotka-Volterra operators for which any order Cesàro mean diverges. This class of Lotka-Volterra operators encompasses all previously presented operators in this context.
\end{abstract}

\section{INTRODUCTION}

A mapping $V: S^{m-1} \rightarrow S^{m-1}, V(x)=x^{\prime}$ such that

$$
x_{k}^{\prime}=\sum_{i, j=1}^{m} p_{i j k} x_{i} x_{j}, \quad \forall k=\overline{1, m}
$$

is called a quadratic stochastic operator, where $p_{i j k}=p_{j i k} \geq 0, \sum_{k=1}^{m} p_{i j k}=1$ for all $i, j, k$ and $S^{m-1}=\left\{x=\left(x_{1}, \cdots, x_{m}\right) \in \mathbb{R}^{m}: x_{i} \geq 0, \sum_{i=1}^{m} x_{i}=1\right\}$ is the $(m-1)$ dimensional standard simplex. Based on some numerical calculations, S.M. Ulam conjectured [7] that the ergodic theorem holds true for any quadratic stochastic

Date: Received: Feb. 20, 2015; Accepted: Jun. 11, 2015.

2010 Mathematics Subject Classification. Primary 47H25; Secondary 47J35.

Key words and phrases. Lotka-Volterra operator, ergodic theorem, Cesàro mean. 
operator $V$, that is the limit

$$
\lim _{n \rightarrow \infty} \frac{1}{n} \sum_{i=0}^{n-1} V^{(i)}(x)
$$

exists for any $x \in S^{m-1}$ where $V^{(i+1)}=V \circ V^{(i)}$. However, Zakharevich showed [8] that Ulam's conjecture is false in general. He showed that the limit (1.1) does not exist for the following quadratic stochastic operator $V: S^{2} \rightarrow S^{2}$ for any $x \in \operatorname{Int} S^{2} \backslash\left\{\left(\frac{1}{3}, \frac{1}{3}, \frac{1}{3}\right)\right\}$ where $V(x)=x^{\prime}$ and

$$
x_{1}^{\prime}=x_{1}^{2}+2 x_{1} x_{2}, \quad x_{2}^{\prime}=x_{2}^{2}+2 x_{2} x_{3}, \quad x_{3}^{\prime}=x_{3}^{2}+2 x_{1} x_{3} .
$$

Some years later, Ganikhodjaev and Zanin [2] have generalized Zakharevich's example in the class of quadratic stochastic Volterra operators acting on 2D simplex. Namely they proved that the limit (1.1) does not exist for the following quadratic stochastic operator $V_{a, b, c}: S^{2} \rightarrow S^{2}$ for any $x \in \operatorname{Int} S^{2} \backslash\left\{P_{0}\right\}$ where $V_{a, b, c}(x)=x^{\prime}$

$$
x_{1}^{\prime}=x_{1}\left(1+a x_{2}-b x_{3}\right), \quad x_{2}^{\prime}=x_{2}\left(1-a x_{1}+c x_{3}\right), \quad x_{3}^{\prime}=x_{3}\left(1+b x_{1}-c x_{2}\right)
$$

and $P_{0}=\left(\frac{c}{a+b+c}, \frac{b}{a+b+c}, \frac{a}{a+b+c}\right)$ with nonzero parameters $a, b, c \in[-1,1]$ having the same sign. If one has that $a=b=c=1$ then $V_{1,1,1}$ is Zakharevich's example given by $(1.2)$.

We define the $k$-th order Cesàro mean by the following formula

$$
C e s_{k}^{(n)}(x, V)=\frac{1}{n} \sum_{i=0}^{n-1} \operatorname{Ces}_{k-1}^{(i)}(x, V), \quad k \in \mathbb{N}
$$

and $C e s_{0}^{(i)}(x, V)=V^{(i)}(x)$.

It is clear that the first order Cesàro mean $C e s_{1}^{(n)}(x, V)$ is nothing but $\frac{1}{n} \sum_{i=0}^{n-1} V^{(i)}(x)$. Based on these notations, the previous results say that the first order Cesàro mean $\left\{C e s_{1}^{(n)}\left(x, V_{a, b, c}\right)\right\}_{n=0}^{\infty}$ of the operator $V_{a, b, c}$ given by (1.3) diverges for any $x \in \operatorname{Int} S^{2} \backslash\left\{P_{0}\right\}$. Surprisingly, it was proven in [6] that any order Cesàro mean $\left\{\operatorname{Ces}_{k}^{(n)}\left(x, V_{a, b, c}\right)\right\}_{n=0}^{\infty}$ of the operator $V_{a, b, c}$ diverges for any $x \in \operatorname{Int} S^{2} \backslash\left\{P_{0}\right\}$. The reader may refer to $[3,5]$ for the resent development of this subject.

A mapping $V_{\mathbf{F}}: S^{m-1} \rightarrow S^{m-1} V_{\mathbf{F}}(x)=x^{\prime}$ such that

$$
x_{k}^{\prime}=x_{k}\left(1+f_{k}(x)\right), \quad k=\overline{1, m}
$$

is called a Lotka-Volterra operator where the mapping $\boldsymbol{F} \equiv\left(f_{1}, f_{2}, \ldots, f_{m}\right)$ : $S^{m-1} \rightarrow \mathbb{R}^{m}$ satisfies the following conditions:

$1^{0}$ The mapping $\boldsymbol{F}$ is continuous;

$2^{0}$ One has that $f_{k}(x) \geq-1$ for all $x \in S^{m-1}$ and $k=\overline{1, m}$;

$3^{0}$ One has that $\sum_{k=1}^{m} x_{k} f_{k}(x)=0$ for all $x \in S^{m-1}$;

$4^{0}$ For every $\alpha \subset\{1,2, \cdots, m\}$ one has that $f_{k}(x)>-1, \forall x \in \operatorname{Int} \Gamma_{\alpha}, k \in \alpha$. 
where $\Gamma_{\alpha}=\operatorname{conv}\left\{e_{k}\right\}_{k \in \alpha}$ and $\left\{e_{k}\right\}_{i=1}^{m}$ is the standard basis of $\mathbb{R}^{m}$.

A Lotka-Volterra operator is a discrete analogy of a generalized predator-prey model. It is worth of mentioning that a quadratic stochastic operator is the Lotka-Volterra operator if and only if $p_{i j k}=0$ whenever $k \notin\{i, j\}$.

In this paper, we present a new class of Lotka-Volterra operators (not quadratic stochastic operator) defined on the two dimensional simplex for which the ergodic theorem will fail.

\section{Non-Ergodic LotkA-Volterra Operators}

Let $f: S^{2} \rightarrow[-1,1]$ be any $C^{1}$-smooth functional (having the first order continuous partial derivatives). We define the Lotka-Volterra operator $V_{f}: S^{2} \rightarrow$ $S^{2}, V_{f}(x, y, z)=\left(x^{\prime}, y^{\prime}, z^{\prime}\right)$ as follows

$$
V_{f}:\left\{\begin{array}{l}
x^{\prime}=x[1+(a y-b z) f(x, y, z)] \\
y^{\prime}=y[1+(c z-a x) f(x, y, z)] \\
z^{\prime}=z[1+(b x-c y) f(x, y, z)]
\end{array}\right.
$$

where $a, b, c \in[-1,1]$.

It is clear that if $f \equiv$ const then the Lotka-Volterra operator $V_{f}$ is the quadratic stochastic operator given by (1.3).

Throughout this paper, we always assume that the non-zero parameters $a, b, c$ have the same sign and $f: S^{2} \rightarrow[-1,1]$ is a non-vanishing $C^{1}$-smooth functional. Moreover, we present the proofs of all results in the case $a, b, c>0$ and $f: S^{2} \rightarrow$ $[m, M]$ where $0<m \leq M \leq 1$. In other cases, the proofs are similar.

It is easy to check that the fixed points of $V_{f}$ are only points $e_{1}=(1,0,0), e_{2}=$ $(0,1,0), e_{3}=(0,0,1)$, and $P_{0}=\left(\frac{c}{a+b+c}, \frac{b}{a+b+c}, \frac{a}{a+b+c}\right)$. Moreover, $P_{0}$ is repelling and $e_{1}, e_{2}, e_{3}$ are saddle points. The trajectory of $V_{f}$ starting from any initial point $P \in \partial S^{2} \backslash\left\{e_{1}, e_{2}, e_{3}\right\}$ moves along the boundary of the simplex as $e_{1} \rightarrow$ $e_{3} \rightarrow e_{2} \rightarrow e_{1}$.

Lemma 2.1. The omega limiting set $\omega(P)$ of the trajectory of $V_{f}$ starting from any initial point $P \in$ Int $^{2} \backslash\left\{P_{0}\right\}$ is infinite and lies on the boundary $\partial S^{2}$ of the simplex $S^{2}$.

Proof. We set $P=(x, y, z), \varphi(P)=x^{c} y^{b} z^{a}$ and

$$
\psi(P)=[1+(a y-b z) f(P)]^{c}[1+(c z-a x) f(P)]^{b}[1+(b x-c y) f(P)]^{a} .
$$

Due to Young's inequality, we get that

$$
\psi(P) \leq\left(1+\frac{c(a y-b z) f(P)+b(c z-a x) f(P)+a(b x-c y) f(P)}{a+b+c}\right)^{a+b+c}=1 .
$$

On the other hand, we obtain that

$$
\varphi\left(V_{f}^{(n+1)}(P)\right)=\varphi\left(V_{f}^{(n)}(P)\right) \psi\left(V_{f}^{(n)}(P)\right) \leq \varphi\left(V_{f}^{(n)}(P)\right),
$$

i.e. $\left\{\varphi\left(V_{f}^{(n)}(P)\right)\right\}_{n=0}^{\infty}$ is a decreasing sequence and converges to some limit $\lambda$. Since $P \neq P_{0}$, we have that $0 \leq \lambda<\varphi(P)<\varphi\left(P_{0}\right)$. 
If $\lambda>0$ then $1=\lim _{n \rightarrow \infty} \frac{\varphi\left(V_{f}^{(n+1)}(P)\right)}{\varphi\left(V_{f}^{(n)}(P)\right)}=\lim _{n \rightarrow \infty} \psi\left(V_{f}^{(n)}(P)\right) \leq 1$. Consequently, for any $P^{*} \in \omega(P)$, one has that $1=\psi\left(P^{*}\right)=\max \psi(P)$, i.e, $P^{*}=P_{0}$. However, this contradicts to $\varphi\left(P^{*}\right)=\lambda<\varphi\left(P_{0}\right)$. It shows that $\lambda=0$, i.e.,

$$
\omega(P) \subset \partial S^{2}=\left\{x \in S^{2}: x_{1} x_{2} x_{3}=0\right\} .
$$

Obviously, $\omega(P) \neq\left\{e_{1}, e_{2}, e_{3}\right\}$. Since $V_{f}(\omega(P))=\omega(P)$, the set $\left\{V_{f}^{(n)}\left(P^{*}\right)\right\}_{n=0}^{\infty} \subset$ $\omega(P)$ for $P^{*} \in \omega(P) \backslash\left\{e_{1}, e_{2}, e_{3}\right\}$ is infinite and so is also $\omega(P)$. This completes the proof.

Let us introduce the following subsets of the simplex: $G_{1}=\left\{\frac{x}{c} \geq \frac{y}{b} \geq \frac{z}{a}\right\}$, $G_{2}=\left\{\frac{x}{c} \geq \frac{z}{a} \geq \frac{y}{b}\right\}, G_{3}=\left\{\frac{z}{a} \geq \frac{x}{c} \geq \frac{y}{b}\right\}, G_{4}=\left\{\frac{z}{a} \geq \frac{y}{b} \geq \frac{x}{c}\right\}, G_{5}=\left\{\frac{y}{b} \geq \frac{z}{a} \geq \frac{x}{c}\right\}$, $G_{6}=\left\{\frac{y}{b} \geq \frac{x}{c} \geq \frac{z}{a}\right\}$. The notation $G_{i} \hookrightarrow G_{j}$ stands for $V_{f}\left(G_{i}\right) \subset G_{i} \cup G_{j}$.

Lemma 2.2. In a long run time, the trajectory of $V_{f}$ moves along the periodic itinerary $G_{1} \hookrightarrow G_{2} \hookrightarrow G_{3} \hookrightarrow G_{4} \hookrightarrow G_{5} \hookrightarrow G_{6} \hookrightarrow G_{1}$.

Proof. Let us show that $G_{1} \hookrightarrow G_{2}$. The rest is similar to this case. Since $\omega(P) \subset$ $\partial S^{2}$ for any $P \in \operatorname{Int} S^{2} \backslash\left\{P_{0}\right\}$, in a long run time, one has that $V_{f}^{(n)}(P) \in \partial S_{\varepsilon}^{2}=$ $\left\{P \in S^{2}: \operatorname{dist}\left(P, \partial S^{2}\right)<\varepsilon\right\}$ for sufficiently small $\varepsilon$. So, we want to show that $V_{f}\left(G_{1} \cap \partial S_{\varepsilon}^{2}\right) \subset G_{1} \cup G_{2}$.

Obviously, for $P=(x, y, z) \in G_{1} \cap \partial S_{\varepsilon}^{2}$, one has that $z<\varepsilon$. Since $a y \geq b z$ and $a x \geq c z$, one has that $\frac{x^{\prime}}{c} \geq \frac{x}{c} \geq \frac{y}{b} \geq \frac{y^{\prime}}{b}$. On the other side, we have that

$$
\frac{z^{\prime}}{a} \leq \frac{2 z}{a} \leq \frac{2 \varepsilon}{a} \leq \frac{\min \{a, b, c\}}{(a+b+c) c} \leq \frac{x}{c} \leq \frac{x^{\prime}}{c}
$$

here, $\varepsilon$ is sufficiently small, say $\varepsilon<\frac{\min \{a, b, c\}}{2(a+b+c)} \min \left\{\frac{a}{b}, \frac{b}{a}, \frac{a}{c}, \frac{c}{a}, \frac{b}{c}, \frac{c}{b}\right\}$, which is suitable for all cases. This means that $\frac{x^{\prime}}{c} \geq \max \left\{\frac{y^{\prime}}{b}, \frac{z^{\prime}}{a}\right\}$, i.e., $V_{f}(P)=P^{\prime} \in G_{1} \cup G_{2}$. This completes the proof.

Let us choose a neighborhood $U_{0}$ of $P_{0}$ such that $U_{0} \subset$ Int $S^{2}$ and $U_{1}=\left(G_{1} \cup\right.$ $\left.G_{2}\right) \backslash U_{0}, U_{2}=\left(G_{3} \cup G_{4}\right) \backslash U_{0}, U_{3}=\left(G_{5} \cup G_{6}\right) \backslash U_{0}$ are convex sets which satisfy $U_{1} \cap U_{2} \cap U_{3}=\emptyset$.

Lemma 2.3. Let $P \notin U, V_{f}^{(k)}(P) \in U$ for all $k=\overline{1, n}$ and $V_{f}^{(n+1)}(P) \notin U$, where $U$ is one of the sets $U_{i}, i=1,2,3$ and $P_{0} \neq P \in I n t S^{2}$. Then, $n>A \log \frac{B}{\varphi\left(V_{f}(P)\right)}$ where $A, B$ are some positive constants.

Proof. Let $P=(x, y, z)$ and $V_{f}^{(k)}(P)=\left(x_{k}, y_{k}, z_{k}\right)$. Without loss of generality, we suppose that $U=U_{1}$. Since $P \notin U_{1}$, one has that $P \in G_{6}$, or equivalently, $\frac{y}{b} \geq \frac{x}{c} \geq \frac{z}{a}$. Since $V_{f}(P) \in U_{1}$, one has that $V_{f}(P) \in G_{1}$, or equivalently, $\frac{x_{1}}{c} \geq$ $\frac{y_{1}}{b} \geq \frac{z_{1}}{a}$. Since $V_{f}^{(n+1)}(P) \notin U_{1}$, one has that $V_{f}^{(n+1)}(P) \in G_{3}$, or equivalently, $\frac{z_{n+1}}{a} \geq \frac{x_{n+1}}{c} \geq \frac{y_{n+1}}{b}$. Therefore, $y \geq \alpha, x_{1} \geq \alpha$, and $z_{n+1} \geq \alpha$ where $\alpha=\frac{\min \{a, b, c\}}{a+b+c}$. We then obtain that

(i) $y_{1}=y[1+(c z-a x) f(P)]=(1-a f(P)) x y+y^{2}+(1+c f(P)) y z \geq y^{2} \geq \alpha^{2}$;

(ii) $\frac{z_{n+1}}{z_{1}}=\prod_{k=1}^{n} \frac{z_{k+1}}{z_{k}}=\prod_{k=1}^{n}\left[1+\left(b x_{k}-c y_{k}\right) f\left(x_{k}, y_{k}, x_{k}\right)\right] \leq 2^{n}$; 
(iii) $\frac{z_{n+1}}{z_{1}} \geq \frac{\alpha}{z_{1}}=\left(\frac{\alpha^{a}}{z_{1}^{a}}\right)^{1 / a}=\left(\frac{x_{1}^{c} y_{1}^{b} \alpha^{a}}{\varphi\left(V_{f}(P)\right)}\right)^{1 / a} \geq\left(\frac{\alpha^{a+2 b+c}}{\varphi\left(V_{f}(P)\right)}\right)^{1 / a}$;

(iv) $2^{n} \geq\left(\frac{\alpha^{a+2 b+c}}{\varphi\left(V_{f}(P)\right)}\right)^{1 / a}$, or equivalently, $n>A \log \frac{B}{\varphi\left(V_{f}(P)\right)}$ for some constants $A, B$. This completes the proof.

Lemma 2.4. Let $U$ be one of the sets $U_{i}, i=1,2,3$ and $P_{0} \neq P \in$ Int $S^{2}$. Let $\left(n_{i}, m_{i}\right)_{i=1}^{\infty}$ be a sequence of natural numbers such that $V_{f}^{\left(n_{i}\right)}(P) \notin U, V_{f}^{\left(n_{i}+k\right)}(P) \in$ $U$ for $k=\overline{1, m_{i}}$, and $V_{f}^{\left(n_{i}+m_{i}+1\right)}(P) \notin U$. Then there exists a constant $c$ such that $m_{i}>c n_{i}$.

Proof. Due to Lemma 2.1 and 2.2, one has that $n_{i} \rightarrow \infty$. Let $\rho=\max _{P \in S^{2} \backslash U_{0}} \psi(P)$. It is clear that $\rho<1$. Then, $\varphi\left(V_{f}(P)\right)=\varphi(P) \psi(P)<\rho \varphi(P)$ for any $P \in S^{2} \backslash U_{0}$. Due to Lemma 2.3, we have that $m_{i}>A \log \frac{B}{\varphi\left(V_{f}^{\left(n_{i}\right)}(P)\right)}>A^{\prime} \log \frac{B}{\rho^{n_{i}} \varphi\left(P_{0}\right)}>c n_{i}$

Corollary 2.5. Let $U_{1 \varepsilon}, U_{2 \varepsilon}, U_{3 \varepsilon}$ be a sufficiently small disjoint neighborhoods of the vertices of the simplex $S^{2}$ and $U_{\varepsilon}=U_{1 \varepsilon} \cup U_{2 \varepsilon} \cup U_{3 \varepsilon}$. Let $\Lambda_{n}=U_{\varepsilon} \cap\left\{V_{f}^{(k)}(x)\right\}_{k=0}^{n}$ and $\#\left(\Lambda_{n}\right)$ be a number of elements of $\Lambda_{n}$. Then one has that $\lim _{n \rightarrow \infty} \frac{\#\left(\Lambda_{n}\right)}{n}=1$.

Let $\Upsilon\left(x, V_{f}: U\right)$ be a function such that

$$
\Upsilon\left(x, V_{f}: U\right)=\left\{\begin{array}{lll}
1 & \text { if } & V_{f}(x) \in U \\
0 & \text { if } & V_{f}(x) \notin U
\end{array}\right.
$$

Let $U_{1 \varepsilon}, U_{2 \varepsilon}, U_{3 \varepsilon}$ be a sufficiently small disjoint neighborhoods of the vertices of the simplex $S^{2}$ and $U$ be one of the sets $U_{i \varepsilon}, i=1,2,3$. Suppose that $x \in U$. We define the following sequence $\left\{\Upsilon\left(x, V_{f}^{(n)}: U\right)\right\}_{n=0}^{\infty}$, i.e.,

$$
\underbrace{1,1, \cdots, 1}_{p_{1}}, \underbrace{0,0, \cdots, 0}_{q_{1}}, \underbrace{1,1, \cdots, 1}_{p_{2}}, \underbrace{0,0, \cdots, 0}_{q_{2}}, \cdots
$$

Corollary 2.6. Let $\left\{p_{n}, q_{n}\right\}_{n=1}^{\infty}$ be a sequence defined above. There exists a constant c mentioned in Lemma 2.4 such that for any $n \geq 2$ one has that

$$
p_{n}>c \sum_{i=1}^{n-1}\left(p_{i}+q_{i}\right), \quad q_{n}>c\left(\sum_{i=1}^{n-1}\left(p_{i}+q_{i}\right)+p_{n}\right) .
$$

Theorem 2.7. Let $V_{f}: S^{2} \rightarrow S^{2}$ be the Lotka-Volterra operator given by (2.1). If non-zero parameters $a, b, c$ have the same sign and $f: S^{2} \rightarrow[-1,1]$ is a non-vanishing $C^{1}$-smooth functional then the limit (1.1) does not exist for any $x \in \operatorname{Int} S^{2} \backslash\left\{P_{0}\right\}$.

Proof. Let us assume that $\lim _{n \rightarrow \infty} \frac{1}{n} \sum_{k=0}^{n-1} V_{f}^{(k)}(P)=P^{*}$ for any $P \in$ Int $S^{2}$ such that $P \neq P_{0}$. Suppose that $P^{*} \notin U_{1}$ and $\left(n_{i}, m_{i}\right)$ are as in Lemma 2.4. Let 
$\delta=\operatorname{dist}\left(P^{*}, U_{1}\right)$ and $\lambda_{i}=\frac{m_{i}}{n_{i}}$. Then $\lambda_{i}>c$ and $\operatorname{dist}\left(\frac{1}{n} \sum_{k=0}^{n-1} V_{f}^{(k)}(P), P^{*}\right)<\frac{\delta}{K}$ for sufficiently large $K$. We let

$$
P^{\prime}=\frac{1}{1+\lambda_{i}}\left(\frac{1}{n_{i}} \sum_{k=0}^{n_{i}-1} V_{f}^{(k)}(P)\right)+\frac{\lambda_{i}}{1+\lambda_{i}}\left(\frac{1}{m_{i}} \sum_{k=n_{i}}^{n_{i}+m_{i}-1} V_{f}^{(k)}(P)\right) .
$$

Then, we have that

$$
\begin{aligned}
\operatorname{dist}\left(P^{\prime}, P^{*}\right) & \geq \operatorname{dist}\left(\frac{1}{n_{i}} \sum_{k=0}^{n_{i}-1} V_{f}^{(k)}(P), P^{\prime}\right)-\frac{\delta}{K} \\
& =\frac{\lambda_{i}}{1+\lambda_{i}} \operatorname{dist}\left(\frac{1}{n_{i}} \sum_{k=0}^{n_{i}-1} V_{f}^{(k)}(P), \frac{1}{m_{i}} \sum_{k=n_{i}}^{n_{i}+m_{i}-1} V_{f}^{(k)}(P)\right)-\frac{\delta}{K} \\
& \geq \frac{c}{1+c} \operatorname{dist}\left(\frac{1}{n_{i}} \sum_{k=0}^{n_{i}-1} V_{f}^{(k)}(P), U_{1}\right)-\frac{\delta}{K} \\
& \geq \frac{c}{1+c}\left(\operatorname{dist}\left(P^{*}, U_{1}\right)-\frac{\delta}{K}\right)-\frac{\delta}{K} \\
& \geq\left(\frac{c}{1+c}-\frac{1}{K} \frac{2+c}{1+c}\right) \delta>\frac{\delta}{K}
\end{aligned}
$$

for sufficiently large $n$. This is a contradiction. This completes the proof

It turns out that any order Cesàro mean $\left\{\operatorname{Ces}_{k}^{(n)}\left(x, V_{f}\right)\right\}_{n=0}^{\infty}$ of the LotkaVolterra operator $V_{f}$ diverges for any $x \in \operatorname{Int} S^{2} \backslash\left\{P_{0}\right\}$. For that purpose, we need one auxiliary result which was proven in [6].

Lemma 2.8. Let $\left\{1^{p_{n}} 0^{q_{n}}\right\}_{n=1}^{\infty}$ be a sequence defined by (2.2) in which $p_{n}, q_{n}$ satisfy the inequality (2.3). Then any order Cesàro mean of the sequence $\left\{1^{p_{n}} 0^{q_{n}}\right\}_{n=1}^{\infty}$ diverges.

Proof. For the sake of completeness, we present the proof of this technical lemma in the case when the sequence $\left\{p_{n}, q_{n}\right\}_{n=1}^{\infty}$ generates a geometric progression $\left\{q^{n}\right\}_{n=0}^{\infty}$ for some integer $q$. In the general case, the technique is the same.

Consequently, we want to show that any (say $k$ ) order Cesàro mean of the sequence $\left\{a_{n}\right\}_{n=0}^{\infty}=\left\{1^{q^{n}} 0^{q^{n+1}}\right\}_{n=0}^{\infty}$ diverges. To do so, it is enough to show that the sequence $\left\{\gamma_{n}^{(k)}\right\}_{n=0}^{\infty}$ diverges (see $[1,4]$ ), where

$$
\gamma_{n}^{(k)}=\frac{C_{n+k-1}^{k-1} a_{0}+C_{n+k-2}^{k-1} a_{1}+\cdots+C_{k-1}^{k-1} a_{n}}{C_{n+k}^{k}}
$$

and $C_{n}^{m}=\frac{n !}{m !(n-m) !}$ is the binomial coefficient. It is worth of mentioning that

$$
C_{n+k}^{k}=C_{n+k-1}^{k-1}+C_{n+k-2}^{k-1}+\cdots+C_{k-1}^{k-1} .
$$

We shall consider two subsequences of the sequence $\left\{\gamma_{n}^{(k)}\right\}_{n=0}^{\infty}$. 
CASE I. Let $n=1+q+\cdots+q^{2 m-1}=\frac{q^{2 m}-1}{q-1}$. We then obtain that

$$
\gamma_{n}^{(k)}=\frac{C_{n+k-1}^{k-1}+\sum_{i=1}^{m-1}\left[C_{n+k-\frac{q^{2 i}-1}{q-1}}^{k}-C_{n+k-\frac{q^{2 i+1}-1}{q-1}}^{k}\right]}{C_{n+k}^{k}}=\frac{C_{n+k-1}^{k-1}}{C_{n+k}^{k}}+\beta_{n}^{(k)}
$$

Since $\lim _{n \rightarrow \infty} \frac{C_{n+k-1}^{k-1}}{C_{n+k}^{k}}=0$, it is enough to study the sequence $\left\{\beta_{n}^{(k)}\right\}_{n=0}^{\infty}$.

Let $r_{i}=\frac{q^{2 i}-1}{q-1}$ and $r_{i}^{\prime}=\frac{q^{2 i+1}-1}{q-1}$. It is easy to check that

$$
\beta_{n}^{(k)}=\frac{\sum_{i=1}^{m-1}\left[\left(n-r_{i}+1\right) \cdots\left(n-r_{i}+k\right)-\left(n-r_{i}^{\prime}+1\right) \cdots\left(n-r_{i}^{\prime}+k\right)\right]}{(n+1) \cdots(n+k)}
$$

Let $p_{i}=q^{2 i}$ and $\delta_{j}=n-r_{i}+j$ for $j=\overline{1, k}$. Since $r_{i}^{\prime}=r_{i}+p_{i}$, we get that

$$
\begin{aligned}
& \left(n-r_{i}+1\right) \cdots\left(n-r_{i}+k\right)-\left(n-r_{i}^{\prime}+1\right) \cdots\left(n-r_{i}^{\prime}+k\right)= \\
& =(-1)^{k+1}\left[p_{i}^{k}-\left(\sum_{j} \delta_{j}\right) p_{i}^{k-1}+\cdots+(-1)^{k-1}\left(\sum_{j_{1} \ldots j_{k-1}} \delta_{j_{1}} \cdots \delta_{j_{k-1}}\right) p_{i}\right]
\end{aligned}
$$

We know that

$$
\lim _{m \rightarrow \infty} \frac{1}{n^{k}} \sum_{i=1}^{m-1} p_{i}^{k}=\frac{(q-1)^{k}}{q^{2 k}-1}, \quad \lim _{m \rightarrow \infty} \frac{1}{n^{l+r}} \sum_{i=1}^{m-1} p_{i}^{l}=0 .
$$

Moreover, it is easy to check that

$$
\sum_{j_{1} \ldots j_{t}} \delta_{j_{1}} \cdots \delta_{j_{t}}=C_{k}^{t}\left(n-r_{i}\right)^{t}+o\left(n^{t}\right)
$$

Consequently, after some algebraic manipulations, we obtain that

$$
\lim _{m \rightarrow \infty} \gamma_{n}^{(k)}=\Gamma_{1}^{(k)} \equiv(-1)^{k+1} \sum_{t=0}^{k-1} \frac{(-1)^{t} C_{k}^{t}}{q^{k-t}+1} .
$$
that

CASE II. Let $n=1+q+\cdots+q^{2 m}=\frac{q^{2 m+1}-1}{q-1}$. In the similar manner, we obtain

$$
\lim _{m \rightarrow \infty} \gamma_{n}^{(k)}=\Gamma_{2}^{(k)} \equiv(-1)^{k+1} \sum_{t=0}^{k-1} \frac{(-1)^{t} q^{k-t} C_{k}^{t}}{q^{k-t}+1}
$$

It is clear that $\Gamma_{1}^{(k)}+\Gamma_{2}^{(k)}=1$ for any $k$. Moreover, it is easy to check that $\Gamma_{1}^{(k)} \neq \frac{1}{2}$ and $\Gamma_{2}^{(k)} \neq \frac{1}{2}$. Therefore, $\Gamma_{1}^{(k)} \neq \Gamma_{2}^{(k)}$ for any $k$. This completes the proof.

Lemma 2.8 immediately implies the following result which is the generalization of Theorem 2.7.

Theorem 2.9. Let $V_{f}: S^{2} \rightarrow S^{2}$ be the Lotka-Volterra operator given by (2.1). If non-zero parameters $a, b, c$ have the same sign and $f: S^{2} \rightarrow[-1,1]$ is a nonvanishing $C^{1}$-smooth functional then any order Cesàro mean defined by (1.4) of the Lotka-Volterra operator $V_{f}$ diverges for any $x \in \operatorname{Int} S^{2} \backslash\left\{P_{0}\right\}$. 
Acknowledgement. The author wishes to thank Junior Associateship Scheme, the Abdus Salam International Centre for Theoretical Physics (ICTP), Trieste, Italy, where this paper was finished, for the invitation and hospitality.

\section{REFERENCES}

1. G.M. Fikhtengolts, A course of differential and integral calculus Vol. II, Moscow, 2001.

2. N.N. Ganikhodzhaev and D.V. Zanin, On a necessary condition for the ergodicity of quadratic operators defined on the two-dimensional simplex, Russian Math Survey 59 (2004), no. 3. (357), 161-162.

3. R.N. Ganikhodzhaev, F.M. Mukhamedov and U.A. Rozikov, Quadratic stochastic operators: Results and open problems, Inf. Dim. Anal. Qua. Prob. Rel. Top. 14 (2011), no 2, 279-335.

4. G.H. Hardy, Divergent Series, Oxford University Press, Great Britain, 1949.

5. F. Mukhamedov and M. Saburov, On homotopy of Volterrian quadratic stochastic operators, Appl. Math Info. Sci. 4 (2010), no. 1, 47-62.

6. M. Saburov, On ergodic theorem for quadratic stochastic operators, Doklady of Uzbek Academy Sci. 6 (2007), 8-11.

7. S.M. Ulam, A collection of mathematical problems, New-York, London, 1960.

8. M.I. Zakharevich, On the behaviour of trajectories and the ergodic hypothesis for quadratic mappings of a simplex, Russian Math Survey 33 (1978), no. 6, 207-208.

Department of Computational \& Theoretical Sciences, Faculty of Science, International Islamic University Malaysia, Jalan Bandar Indera Mahkota, P.O. Box, 25200, Kuantan Pahang, Malaysia.

E-mail address: msaburov@gmail.com; msaburov@iium.edu.my 